\title{
Instrumentos de evaluación de las influencias socioculturales en la interiorización de ideales corporales en población hispana
}

\section{Instruments for the evaluation of sociocultural influences in the internalization of body ideals in the hispanic population}

Rosalia Vázquez-Arevalo', Mariana Valdez Aguilar², Xochitl López Aguilar1 y Juan Manuel Mancilla-Diaz

${ }^{1}$ Universidad Nacional Autónoma de México, FES Iztacala, Grupo de Investigación en Nutrición

${ }^{2}$ Estudiante del Doctorado en Psicología de la Universidad Nacional Autónoma de México

Recibido: 2019-02-13

Revisado: 2019-03-21

Aceptado: 2020-01-13

Autora de correspondencia: rvamap@unam.mx (R. Vázquez)

Financiamiento: PAPIIT IN306518

Abstract The aim of this article was to analyze the use of the Questionnaire of Sociocultural Influences on the Aesthetic Body Shape Model (CIMEC) and The Sociocultural Attitudes Towards Appearance Questionnaire (SATAQ) in Hispanic literature. The background and validations of each questionnaires are presented, as well as examples of their use in spanish-speaking countries. The CIMEC has two long versions of 40 -items, the short version of 17 -items for men and the one of 26 -items for women. Regard the SATAQ five versions were found, the first of 11-items, and the last version of the SATAQ-4R, which is the only version that has a differentiation between men and women and has 31 and 28 items respectively. To analyze the psychometric properties of the instruments, the exploratory and confirmatory factor analysis were used. It is concluded that the CIMEC is the most used instrument in Latin America and it has been worked to reduce the number of items, since its creation it has included the influence of advertising, peers and family, as well as, internalizing the musculature/ thinness; while the SATAQ has increased its items, considering aspects such as peer pressures, family, and muscle/ thinness stereotypes, currently the two questionnaires have very similar contents. 
Keywords: $\quad$ sociocultural influences, aesthetic body model, Hispanic population, SATAQ CIMEC

Resumen El objetivo del presente artículo fue analizar la utilización del Cuestionario de Influencias Socioculturales sobre el Modelo Estético Corporal (CIMEC) y Sociocultural Attitudes Towards Appearance Questionnare (SA$\mathrm{TAQ}$ ) en la literatura hispanoamericana. Se presentan los antecedentes y validaciones de cada uno de los instrumentos, así como ejemplos de su utilización en países de habla hispana. En el CIMEC se presentan sus dos versiones largas de 40 ítems y las cortas 17 ítems para varones y 26 para mujeres. Para el SATAQ se localizaron 5 versiones, la primera de 11 ítems y la última versión del SATAQ-4R-, la cual, es la única que tiene la diferenciación entre hombres y mujeres, y cuenta con 31 y 28 ítems respectivamente. Para analizar las propiedades psicométricas de los instrumentos se han empleado el análisis factorial exploratorio y confirmatorio. Se concluye que el CIMEC es el más utilizado en Hispanoamérica y que se ha trabajado en reducir el número de preguntas, ya que desde su creación ha incluido la influencia de la publicidad, los pares y la familia, así como interiorización la musculatura/ delgadez, mientras que el SATAQ, ha ido aumentado sus ítems, considerando aspectos como las presiones de los pares, familia, y estereotipos de musculatura/delgadez, quedando actualmente los dos instrumentos con contenidos muy semejantes.

Palabras clave: influencias socioculturales, modelo estético corporal, población hispana, SATAQ CIMEC

\section{Introducción}

El interés por la imagen corporal tiene cerca de cien años, ya en 1920, Mead (como se citó en Cash y Pruzinsky, 2002), mencionaba que ésta podría definirse como la "habilidad del cerebro para detectar peso, limitar forma y tamaño e incorporar éstos dentro de los esquemas mentales (pág. 3)”. Dicho interés por la imagen corporal se acentuó en la década de 1970 cuando se observó que la interiorización de un modelo corporal predominante podía estar asociado a psicopatologías como la anorexia y bulimia nerviosas en las mujeres. En el caso de los varones la preocupación generalizada por el cuerpo o cierto tipo de figura es más reciente, hace 25 años aproximadamente; no obstante, también tiene consecuencias graves en algunos casos, como el desarrollo del trastorno dismórfico corporal.

Bajo el concepto de influencias socio-culturales se estudia una serie de factores culturales y sociales que han promovido y validado valores estéticos como la delgadez para mujeres y la musculatura para varones, por lo que es importante la construcción de medidas o instrumentos que valoren este constructo, por ello, el objetivo de este artículo es analizar la utilización del Cuestionario de Influencias Socioculturales sobre el Modelo Estético Corporal (CIMEC) y del Sociocultural Attitudes Towards Appearance Questionnare (SATAQ), en la literatura hispana para medir las influencias culturales de los modelos estéticos

La cultura tiene una relevante influencia en la promoción de modelos corporales ideales, con gran apoyo de los diversos medios masivos de comunicación y las llamadas "redes sociales", ya que las formas corporales son tema de conversación y preocupación cotidiana; no obstante, debemos entender que los individuos no son agentes pasivos, ellos van conformando desde la infancia una idea de belleza, que al parecer tarde o temprano tendrá que ser manifestada en su entorno social y formará parte de su vivencia personal.

Podríamos hablar de cultura corporal para referirnos a las prácticas y costumbres creadas para entender y valorar el cuerpo en una sociedad, la cual se transmite a través del entorno social; la familia, los amigos, así como de los contextos sociales más cercanos. En nuestra cultura occidentalizada, el modelo corporal que prevalece desde hace más de medio siglo es la emaciación para las mujeres, y desde hace por lo 
menos dos décadas, la musculatura para los varones. Estos ideales estéticos no son inicuos, han facilitado el desarrollo de psicopatologías en una proporción de la población, como son anorexia y bulimia nerviosas en el caso de las féminas y dismorfía muscular en el caso de los varones.

La transmisión de la delgadez corporal como un objetivo colectivo a perseguir por las mujeres se puede observar cada día a través de diferentes medios de comunicación masiva, que bombardean a su público con imágenes emaciadas asociadas a la belleza, prestigio social, salud y éxito, esto ha sido ampliamente probado (Stice, Schupak-Neuberg, Shaw y Stein, 1994; Harrison y Hefner, 2006 y Tiggemann, 2014). Stice et al. (1994) encontraron que las mujeres expuestas a imágenes de mujeres delgadas experimentaron mayor interiorización del modelo corporal delgado comparado con el de mujeres que fueron expuestas a imágenes neutras, por tanto, contaban con más probabilidades de sentirse insatisfechas con sus cuerpos.

En el caso de los varones, el estudio de los modelos estéticos es más reciente, no obstante, Leit, Gray y Pope (2002), examinaron el efecto que tenían las imágenes de cuerpos masculinos que aparecían en los medios de comunicación sobre las actitudes de los varones hacia la apariencia corporal, encontrando que los estudiantes expuestos a figuras musculosas habían mostrado una discrepancia significativamente mayor entre la musculatura percibida y la que deseaban tener. Actualmente algunos estudios muestran el efecto que pudieron tener los cambios estéticos en los juguetes y/o los denominados "súper héroes", ya que lo muñecos han tenido transformaciones en las últimas décadas, que los muestra más musculosos (Young, Gabriel y Hollar, 2013) y estos efectos pueden ser nocivos en los varones que presentan insatisfacción corporal (Galioto y Crowther, 2013).

La internalización de los estándares sociales de belleza se refiere a la influencia que los factores socio-culturales pueden tener en la incorporación de valores específicos sobre el cuerpo en una persona y en qué nivel éstos valores pueden llegar a convertirse en la principal finalidad en la vida de un individuo. Una de las teorías que intenta dar cuenta de cómo ocurre esto es el Modelo Tripartita de Influencia (Thompson, Heinberg, Altabe y Tantleff-Dunn, 1999; van den Berg,
Thompson, Obremski-Brandon y Coovert, 2002), que rescata la influencia de los medios de comunicación, la familia y los amigos/iguales.

Con la finalidad de conocer las influencias socioculturales en la interiorización de los modelos estéticos se han creado diferentes instrumentos, dos son los más utilizados en las investigaciones con población hispanoparlante: 1) Cuestionario de Influencias Socioculturales Sobre el Modelo Estético Corporal (CIMEC) y, 2) The Sociocultural Attitudes Towards Appearance Questionnaire (SATAQ).

\section{Cuestionario de Influencias Socioculturales Sobre el Modelo Estético Corporal (CIMEC).}

El CIMEC es un cuestionario con 40 preguntas, tiene dos versiones, masculina y femenina, aunque se publicó primero la de mujeres y quizás es la más difundida porque está relacionada a la anorexia y bulimia nerviosas. Fue elaborado y validado en población española, con estudiantes universitarias, con pacientes con anorexia nerviosa y mujeres controles por Toro, Salamero y Martínez (1994), con el objetivo de explorar la interiorización de las influencias culturales sobre el modelo estético corporal. En su versión para mujeres se propuso un punto de corte $\geq 23-24$ y se determinó una estructura de cinco factores. En México, su validación fue realizada por Vázquez, Álvarez y Mancilla (2000) con una muestra de 1001 estudiantes mujeres, su análisis propuso una estructura de cuatro factores, que explicó $45.3 \%$ de la varianza; el Alfa de la escala total fue de .90 (ver tabla 1 ).

Posteriormente en 2005, Toro, Castro, Gila y Pombo, tuvieron como objetivo crear una versión para varones que fuera más corta, de la que se había creado originalmente (40 preguntas) y que tuviera suficiente poder discriminatorio, con 17 de las 20 preguntas que diferenciaron a los controles de los varones con anorexia obtuvieron el CIMEC-V-17. El instrumento obtuvo un Alfa de .85, lo que indicó una adecuada consistencia interna. Se propuso una estructura con cuatro factores: 1) Influencia de la Información y la Publicidad, 2) Preocupación por Volverse Obeso, 3) Influencia de los Modelos de Musculatura, y 4) Comidas Sociales y Ejercicio (ver tabla 2).

En el caso de México, con la primera versión de 40 preguntas para varones se realizó una validación en 
Tabla 1.- Características psicométricas del CIMEC, en España y México.

\begin{tabular}{|c|c|c|}
\hline Autores, año y país & Participantes & Resultados \\
\hline $\begin{array}{l}\text { Toro, Salamero y Martínez } \\
\text { (1994). España }\end{array}$ & $\begin{array}{l}\text { 1er. Análisis } \\
n=118 \text { jóvenes españolas. } \\
n=59 \text { pacientes con anorexia } \\
n=59 \text { controles. }\end{array}$ & $\begin{array}{l}\text { Factores: } \\
\text { Malestar con la Imagen Corporal } \\
\text { Varianza }=32.9 \% \\
\text { Influencia de la Publicidad } \\
\text { Varianza }=6.1 \% \\
\text { Influencia de los Modelos Sociales } \\
\text { Varianza }=4.5 \% \\
\text { Alfa total }=.93\end{array}$ \\
\hline $\begin{array}{l}\text { Toro, Salamero y Martínez } \\
(1994) \\
\text { España } \\
\text { CIMEC } 26\end{array}$ & $\begin{array}{l}2^{\mathrm{a}} \text { Análisis } \\
n=118 \text { jóvenes españolas. } \\
n=59 \text { pacientes con anorexia } \\
n=59 \text { controles. }\end{array}$ & $\begin{array}{l}\text { Se aislaron } 26 \text { variables mediante un análisis factorial con } \\
\text { altas cargas en cada factor. } \\
\text { Factores: } \\
\text { Malestar por la Imagen Corporal } \\
\text { Influencia de la Publicidad } \\
\text { Influencia de los Mensajes Verbales } \\
\text { Influencia de los Modelos Sociales } \\
\text { Influencia de las Situaciones Sociales } \\
\text { Alfa total }=.94 \\
\text { Sensibilidad }=81.4 \% \\
\text { Especificidad }=55.9 \% \\
\text { Punto de corte }=23-24\end{array}$ \\
\hline $\begin{array}{l}\text { Vázquez, Alvarez y } \\
\text { Mancilla (2000) } \\
\text { México }\end{array}$ & $\begin{array}{l}n=1001 \text { mujeres con un rango de } \\
\text { edad de } 14 \text { a } 33 \text { años } M=19.03 \\
D E=2.56\end{array}$ & $\begin{array}{l}\text { El análisis factorial y la rotación varimax proporcionaron } \\
\text { seis factores. Posteriormente, a partir del análisis factorial } \\
\text { confirmatorio se agruparon en cuatro factores que explicaron } \\
45.3 \% \text { de la varianza total. } \\
\text { Influencia de la Publicidad Alfa }=.92 \\
\text { Malestar por la Imagen Corporal y Conductas para Reducir de } \\
\text { Peso Alfa }=.86 \\
\text { Influencia de los Modelos Sociales Alfa }=.75 \\
\text { Influencia de las Relaciones Sociales Alfa }=.63 \\
\text { Alfa total }=.94\end{array}$ \\
\hline
\end{tabular}

muestra universitaria (ver tabla 2), que contó con 5 factores, un Alfa total de .90 (Vázquez et al., 2010). Y recientemente se probó la versión del CIMEC-V de 17 items, también en estudiantes mexicanos, realizando un factorial confirmatorio, que indica una estructura de 3 factores y buen ajuste (ver tabla 2).

La utilización de las versiones del CIMEC ha sido principalmente en España y México, algunos ejemplos de estudios pueden verse en la tabla 3. En la mayoría de las investigaciones el CIMEC es un instrumento utilizado para asociar la interiorización de los modelos estéticos a la anorexia y bulimia nerviosas (Cruz, Ávila, Velázquez, Castillo y Francis, 2013; Escoto, Camacho y Mancilla, 2010; Hernández, Alves, Arroyo y Basabe, 2012; Vázquez et al., 2005), uno de los factores asociados más relevante es la insatisfacción corporal (Hernández, Alves, Arroyo, y Basabe, 2012; Esnaola, Rodríguez y Goñi, 2010; Cruz et al., 2013).

\section{Cuestionario de actitudes socioculturales hacia la apariencia (SATAQ)}

Con la finalidad conocer el efecto que tienen las influencias socioculturales en la internalización de los estándares de belleza y que están asociados a la insatisfacción corporal, además de la anorexia y la bulimia nerviosas, Heinberg, Thompson y Stormer en 1995, construyeron y validaron un cuestionario que denominaron SATAQ. Para su validación participaron 506 mujeres universitarias de 17 a 35 años en tres estudios. En un inicio se tenían 44 preguntas con cinco opciones 
Tabla 2.- Características psicométricas del CIMEC-V para varones en España y México.

\begin{tabular}{ll} 
Autores, año y país & Participantes \\
\hline Toro, Castro, Gila y Pombo & $n=274$ varones. \\
(2005) & $n=34$ varones adolescentes con \\
España & $\begin{array}{l}\text { anorexia nerviosa. } \\
\text { Versión CIMEC-17 }\end{array}$ \\
& alimentario
\end{tabular}

\section{Resultados}

Se propuso la estructura factorial siguiente:

Influencia de la Publicidad e Información

varianza $=35.9 \%$

Preocupación por Volverse Obeso

varianza $=7.9 \%$

Influencia de los Modelos de Musculatura

varianza $=6.8 \%$

Comidas Sociales y Ejercicio

varianza $=6.1 \%$

Se plantea un punto de corte $=5$, con una especificidad de

$71.2 \%$ y una sensibilidad de $73 \%$.

Alfa $=.80$ (Muestra sin trastorno alimentario)

Alfa $=.88$ (Muestra con trastorno alimentario)

Alfa $=.85$ (Por toda la muestra)

Varianza total explicada $=56.9 \%$

Vázquez et al. (2010).

México

Versión CIMEC-40
Vázquez, Valdez, López, López-Cuautle, Mancilla (manuscrito no publicado) México Versión CIMEC-V 17 $n=504$ varones estudiantes con un promedio de edad de 18 años $(D E=3.49)$
Se decidió una estructura de 5 factores que explicaron el $43.42 \%$ de la varianza total: Influencia de la Publicidad $A l f a=.84$, varianza $=13.15 \%$ Influencia de la Figura de Delgadez Alfa $=.68$, varianza $=6.97 \%$ Malestar con la Imagen Corporal Alfa $=.71$, varianza $=6.57 \%$ Influencia de la Figura Musculosa Alfa $=.69$, varianza $=11.57 \%$ Influencia de las Relaciones sociales Alfa $=.69$, varianza $=5.16 \%$ Alfa total $=.90$

$n=624$ varones estudiantes con un promedio de edad de 17 años

Se decidió una estructura factorial de tres factores que explicaron $47.59 \%$ de la varianza total Influencia de la Publicidad e información Alfa $=.74$, varianza $=32.67 \%$ Influencia de los modelos de musculatura Alfa $=.66$, varianza $=7.95 \%$ Preocupación por volverse obeso Alfa $=.76$, varianza $=6.95 \%$ Alfa total $=.86$.

Análisis factorial confirmatorio: RMSEA $=.047, \mathrm{CFI}=.953, \mathrm{NFI}=.914 \mathrm{TLI}=.936, \mathrm{CFI}=.948$ de respuestas (que iban de totalmente de acuerdo a completamente en desacuerdo), después de varios análisis, se decidió una estructura de dos factores con 14 preguntas, que explicaron $64 \%$ de la varianza: 1 ) Escala de Internalización Alfa $=.88$ (8 preguntas) y 2) Escala de Consciencia Alfa $=.71$ ( 6 preguntas).
En 1997 Cusumano y Thompson propusieron una versión revisada (SATAQ-R) y sugirieron tres aspectos para las influencias socioculturales sobre la apariencia: 1)exposición a los medios, 2) conciencia de las presiones sociales sobre la apariencia e 3) internalización de la presión sociocultural sobre la imagen 
Tabla 3.- Investigaciones que han utilizado el CIMEC en Hispanoamérica.

\begin{tabular}{|c|c|}
\hline Autores, año y País & Muestra \\
\hline $\begin{array}{l}\text { Rivarola y Penna (2006) } \\
\text { México }\end{array}$ & $\begin{array}{l}41 \text { mujeres adolescentes } \\
\text { entre } 12 \text { y } 14 \text { años y } 59 \\
\text { adolescentes entre los } 18 \text { y } \\
20 \text { años de edad }\end{array}$ \\
\hline $\begin{array}{l}\text { Vázquez, López, Alvarez, } \\
\text { Mancilla y Oliva (2006) } \\
\text { México }\end{array}$ & $\begin{array}{l}45 \text { niños con edad } \\
\text { promedio de } 10 \text { años y } 42 \\
\text { jóvenes del sexo masculino } \\
\text { con promedio de edad de } \\
19 \text { años }\end{array}$ \\
\hline $\begin{array}{l}\text { Rodríguez y Cruz (2008) } \\
\text { España y Latinoamérica }\end{array}$ & $\begin{array}{l}403 \text { adolescentes de } \\
\text { educación secundaria } \\
\text { obligatoria del País } \\
\text { Vasco; } 191 \text { españolas y } 212 \\
\text { latinoamericanas }\end{array}$ \\
\hline
\end{tabular}

Alvarez, Franco, López, Mancilla y Vázquez (2009) México

Escoto et al. (2010)

México

Ruiz, Vázquez, Mancilla y Trujillo (2010)

México

Mancilla et al. (2010)

España y México

Jáuregui, Tomillo, Santiago y Bolaños (2011) España

Mancilla et al. (2012) México

Participaron 145 mujeres de entre 12 y 46 años

281 participantes de entre 12 y 14 años de edad (144 mujeres y 137 varones) seleccionados al azar

Participaron 103 mujeres mexicanas y sus padres

438 universitarios españoles (254 mujeres y 184 varones) con edad promedio de 20.79 años y 424 universitarios mexicanos (243 mujeres y 181 varones) con edad promedio de 20.61 años

950 varones de 18 a 25 años (672 en 1998 y 278 en 2008) aspirante a soldado profesional

130 mujeres estudiantes, cuya media de edad fue de

\section{Resultados relacionados a las Influencias Culturales}

Los mensajes socioculturales sobre el modelo estético de la delgadez guardan una estrecha relación con los TCA e imagen corporal.

La sintomatología de TCA fue mayor en los niños que en los jóvenes; no obstante, no se halló en ellos una asimilación considerable de los modelos estéticos corporales de delgadez, a diferencia de los jóvenes, quienes al parecer reciben mayor influencia de la publicidad y del grupo de amigos.

La insatisfacción corporal se relaciona directamente con las influencias socioculturales que contribuyen a la internalización del modelo estético corporal vigente; además, el malestar por la imagen corporal y la influencia que ejercen la publicidad, los mensajes verbales, los modelos y situaciones sociales, generan insatisfacción con el propio cuerpo.

La edad se asoció con mayor interiorización del ideal de delgadez, influencia de la publicidad, las relaciones sociales; insatisfacción corporal; por otro lado, el peso se asoció con más interiorización, insatisfacción y síntomas de TCA. 19.44 años
Se encontró que la presencia de sintomatología de TCA se asocia a menor autoestima y mayor influencia de los modelos estéticos corporales en adolescentes varones y mujeres.

La asimilación del modelo estético y la influencia de las relaciones sociales en el aprecio de la delgadez (hijas y madres) fueron factores de riesgo de los TCA y que el estereotipo de la delgadez está mayormente dictado por el ambiente social.

Los resultados proporcionan apoyo a la hipótesis de que la interiorización del modelo ideal de belleza media la relación entre la presión sociocultural para ser delgado y la insatisfacción corporal; además, mostraron que los estudiantes universitarios con sintomatología de TCA obtuvieron mayores puntuaciones en comparación con los grupos sin sintomatología en cuanto a la puntuación total del CIMEC y sus subescalas.

La influencia del modelo estético corporal, la actividad física y la conducta alimentaria están relacionados con un mayor volumen corporal, además de que la influencia del modelo estético en varones ha aumentado, especialmente la de los amigos y la relacionada con conductas dirigidas a perder peso.

La interiorización del ideal de delgadez medió la relación entre la influencia de pares y la insatisfacción corporal, así como la relación entre la influencia de pares y la alimentación no saludable. Este estudio apoya la hipótesis de que la interiorización del ideal de delgadez es un importante mediador sobre la insatisfacción corporal y la alimentación no saludable en jóvenes mexicanas. 
Tabla 3 (Continuación).- Investigaciones que han utilizado el CIMEC en Hispanoamérica.

Autores, año y País

Franco, Díaz, López, Escoto

y Jaime (2013) México
Muestra

289 mujeres estudiantes

universitarias, con edad

promedio de 20.51 años
Resultados relacionados a las Influencias Culturales

La insatisfacción corporal predijo el riesgo de anorexia nerviosa en interacción con la insatisfacción corporal e influencia de la publicidad, mientras que el índice de masa corporal predijo el riesgo de bulimia nerviosa. Se concluye que la insatisfacción corporal juega un papel relevante en la predicción de riesgo de TCA.
Barrientos, Escoto, Bosques, Enríquez y Juárez (2014) México

Espósito (2015) Argentina
Participaron 467 varones y mujeres (169 usuarios de gimnasio y 298 no usuarios de gimnasio) de 13 a 53 años de edad

314 alumnos (144 varones y 170 mujeres) de entre 15 y 19 años de escuela secundaria básica
Se observó una asociación positiva y significativa entre la interiorización de ideales, la preocupación por la delgadez y por la musculatura, al parecer un deseo en todos los varones y en el grupo de mujeres usuarias de gimnasio, de tener mayor musculatura y poca grasa en el cuerpo.

Se detectó que, a mayor nivel de influencia del patrón de belleza, mayor es el nivel de insatisfacción con la propia imagen. Estos resultados permiten hipotetizar que ambas variables funcionan como factores predisponentes y aportan evidencia a las teorías que consideran los TCA como síndromes ligados a la cultura corporal; para ello añadieron siete preguntas a la versión anterior; cuatro a la escala de Consciencia (Alfa $=.83)$ y tres a la escala de Internalización (Alfa $=.89$ ), quedando una escala de 21 preguntas, pero dividida en dos factores.

Posteriormente en 2004, Thompson, Van den Berg, Roehrig, Guarda y Heinberg propusieron el SATAQ-3, observaron a partir de una revisión de la literatura que la influencia de los medios podría abarcar cuatro aspectos: conciencia, internalización, presión e información, además la influencia podría estar relacionada a deportes, atletismo o ejercicio, y debieran considerarse medios más contemporáneos, como revistas, películas, televisión, etc. Con todo ello se desarrollaron más preguntas, y se adaptaron y revisaron los contenidos en el instrumento, quedando 40 preguntas para la nueva versión. Al final se propusieron cuatro escalas y 30 preguntas (ver tabla 4).

El SATAQ-3 es quizá la versión más traducida y adaptada en diferentes países como Italia, Francia, China, Jordania; y en países de habla hispana ha sido validada en España, Argentina y Brasil (ver tabla 5), siendo este último en el que más estudios encontramos con este instrumento.

Los estudios en Hispanoamérica son principalmente con el SATAQ-3, como puede verse en la tabla

Tabla 4.- Datos psicométricos de la validación del SATAQ-3 (Thompson et al., 2004).

\begin{tabular}{|c|c|c|}
\hline Autores, año y país & Participantes & Resultados \\
\hline \multirow[t]{9}{*}{ Thompson et al. (2004) } & $1^{\circ}$ Estudio & 30 preguntas \\
\hline & Dos muestras independientes de mujeres & en cuatro factores \\
\hline & universitarias & $\begin{array}{l}\text { El análisis factorial indicó los siguientes } \\
\text { factores: }\end{array}$ \\
\hline & 1) 175 mujeres de licenciatura con un rango de & Información Alfa = .96 \\
\hline & edad de 17 a 25 años. & Presión Alfa = .92 \\
\hline & $2^{\circ}$ estudio & Internalización Atlética Alfa = .95 \\
\hline & 195 mujeres de licenciatura con un rango de & Internalización Gen Alfa = .96 \\
\hline & edad de 18 a 22 años, además de una muestra de & Alfa total $=.96$ \\
\hline & comparación de 15 pacientes hospitalizadas. & Total de varianza explicada $65 \%$. \\
\hline
\end{tabular}


Tabla 5. Características psicométricas del SATAQ-3 en algunos países.

\begin{tabular}{|c|c|}
\hline Autores, añoy país & Participantes \\
\hline $\begin{array}{l}\text { Madanat, Hawks y Browns } \\
\text { (2006) Jordania }\end{array}$ & $\begin{array}{l}n=100 \text { Mujeres de } 18 \text { años en } \\
\text { adelante. Mujeres embarazadas } \\
\text { fueron excluidas de la muestra. } \\
\text { Edad media }=33.6 \text { (rango = } 18-60 \\
\text { años) }\end{array}$ \\
\hline $\begin{array}{l}\text { Stefanile, Matera, Nerini y } \\
\text { Pisani (2009) Italia }\end{array}$ & $\begin{array}{l}598 \text { mujeres adolescentes } \\
(\text { Edad media }=15.54, D E=2.03)\end{array}$ \\
\hline $\begin{array}{l}\text { Jackson y Chen (2010) } \\
\text { China }\end{array}$ & $\begin{array}{l}n=719 \text { Varones adolescentes } \\
n=317 \text { varones de séptimo grado } \\
n=402 \text { de décimo grado. } \\
\text { De dos escuelas de China. } \\
\text { La edad promedio fue de } 14.55(D E \\
=1.74) \\
B M I=19.66(D E=3.07)\end{array}$ \\
\hline
\end{tabular}

\section{Resultados}

Se realizó un análisis factorial para validar la información. Los factores presentaron la siguiente información:

Información Alfa = .89

Presión Alfa $=.84$

Internalización-General Alfa $=.88$

Internalización Atlética Alfa $=.82$

Varianza acumulada $=63.5 \%$.

Se utilizó un análisis factorial exploratorio para investigar la estructura factorial de la versión Italiana del SATAQ. Los resultados indicaron cuatro factores:

Información Alfa =.91

Presión Alfa $=.91$

Internalización General Alfa $=.94$

Internalización Atlética Alfa $=.84$

Varianza total explicada $=59.7 \%$

Se realizó un análisis factorial exploratorio inicial con una solución de cuatro factores:

Presión-Internalización General ( $A / f a=.94$, varianza explicada $=43.45 \%)$.

Recursos sobre la Apariencia (Información; Alfa = .92, varianza explicada, 6.77\%).

Presión-Internalización (Atlética) Alfa $=.85$ varianza explicada $=7.86 \%$ ).

Presión para ser delgado $(\mathrm{Alf} a=.77$, varianza explicada $=$ $3.82 \%)$.

Se realizó un análisis factorial confirmatorio en un segundo estudio en donde se midió el ajuste de estos cuatro factores del modelo y los alternativos reflejados en el SATAQ-3 de las muestras de Occidente y de Malasia.

Rousseau, Valls y Chabrol (2010) Francia

Soares, Conti, Caputo y Filgueiras (2015) Brasil
818 adolescentes, de preparatoria y universidad. Con una edad media de 16.8 años.

529 adolescentes, 297 femeninas y 232 varones
El análisis de la estructura mostró cuatro factores que explicaron $58.5 \%$ de la varianza total Internalización General (Alfa $=.92$, varianza explicada $=18 \%)$ Información (Alfa $=.87$, varianza explicada $=16.2 \%$ ) Internalización Atlética, (Alfa = .82, varianza explicada =10\%) Presión $(A l f a=.89$, varianza explicada $=14 \%)$

Se utilizó un análisis factorial exploratorio para investigar la estructura factorial de la versión Brasileña del SATAQ. Los resultados indicaron seis factores:

Información de otras fuentes Alfa $=.81$

Internalización Atlética Alfa $=.76$

Presión Alfa $=.67$

Marcador inverso Alfa $=.72$

Información televisiva Alfa $=.51$

Internalización general por padres Alfa $=.63$

Análisis factorial confirmatorio: $R M S E A=.046, S R M R=.068$, $T L I=.84, C F I=.86$ 
Tabla 5 (Continuación). Características psicométricas del SATAQ-3 en algunos países.

Autores, año y país

Murawski, Elizathe, Custodio y Rutsztein (2015)

Argentina

\section{Participantes}

Se incluyó a 58 pacientes mujeres

con TCA y 237 mujeres estudiantes

universitarias de Buenos Aires,

Argentina de entre 18 y 34 años de edad.

\section{Resultados}

Varianza explicada total $=58 \%$ (mujeres con trastorno

alimentario; TA)

$61 \%$ (mujeres sin trastorno alimentario; NTA)

Alfa $=.91 \mathrm{TA}$

Alfa $=.94$ NTA

Información TA Alfa $=.87$ NTA Alfa $=.90$

Presión TA Alfa $=.89$ NTA Alfa $=.92$

Internalización-Ceneral TA Alfa $=.90$ NTA Alfa $=.93$

Internalización-Atlética TA Alfa $=.83$ NTA Alfa $=.82$
6. Argentina, España y Brasil, son los países que más lo refieren en sus estudios, especialmente los dos últimos, al igual que el CIMEC, su finalidad es conocer las relaciones de las influencias socioculturales en relación a la insatisfacción corporal, o variables relacionadas a los trastornos como anorexia o bulimia nerviosas, aunque ha sido usado mayormente en mujeres jóvenes, también hay estudios como adolescentes (Dunker, Fernandes y Carreira, 2009), y deportistas (Mockdece, Fernandes, Berbert, Sousa

\section{Tabla 6. Investigaciones que han utilizado el SATAQ-3 en Hispanoamérica}

\begin{tabular}{ll} 
Autores, año y país & Participantes \\
\hline $\begin{array}{l}\text { Dunker, Fernandes y } \\
\text { Carreira, (2009) Brasil }\end{array}$ & $\begin{array}{l}\text { 183 niñas (entre 15 y 18 años de } \\
\text { edad) de escuelas públicas y } \\
\text { privadas. } \\
\end{array}$ \\
SATAQ-3. \\
Magallares (2013) España & $\begin{array}{l}\text { 375 mujeres de } 18 \text { a } 35 \text { años. } \\
\text { SATAQ-3. }\end{array}$
\end{tabular}

Amaya et al. (2013) México 144 mujeres y 138 varones, entre 9-12 y 13-15 años. SATAQ

Fortes et al. (2015) Brasil

Mockdece, Fernandes, Berbert, Sousa y Caputo (2016) Brasil

Magallares (2016) España
383 varones entre 12 y 17 años. SATAQ-3.

413 adolescentes (varones y mujeres) entre 10 y 18 años. 40 deportistas de élite. 245 atletas de no élite y 128 adolescentes no atletas. SATAQ-3.

450 estudiantes varones de 18 a 29 años.

SATAQ-3.

\section{Resultados}

En cuanto a las influencias socioculturales, fue más alto la interiorización del cuerpo delgado en las escuelas de ámbito privado que público; no obstante, la sobre-estimación corporal fue semejante en ambos grupos.

La comparación social está directa e indirectamente (a través de la ansiedad social) relacionada de forma positiva con el riesgo de desarrollar TCA. Mientras que las actitudes socioculturales sobre la apariencia lo hacen de forma indirecta (a través de la ansiedad social) y positiva con los problemas de conducta alimentaria.

La edad es un predictor de conductas alimentarias anómalas en los varones, mientras que la interiorización del ideal corporal es el principal predictor de insatisfacción corporal en ambos sexos.

La internalización se asoció a conductas restrictivas entre varones jóvenes.

Los factores de riesgo de trastorno alimentario están fuertemente relacionados a la insatisfacción corporal entre deportistas de elite y no-elite.

Los participantes que puntuaron alto en ideología de género informaban de mayores actitudes antiobesos y menor interiorización de la delgadez, preocupación por la comida e insatisfacción corporal 
y Caputo, 2016), pero su utilización es más reciente, podríamos decir que en los últimos cinco años.

En 2015 fue revisado el SATAQ-3, ya que se habían detectados limitaciones conceptuales en cuatro regiones de Estados Unidos y en tres muestras internacionales de mujeres. Surge el SATAQ-4 (Schaefer et al., 2015), donde después de un análisis de los reactivos ya existentes, se prueban 51 preguntas en dos grandes rubros: internalización de los modelos estéticos (27 preguntas) y presión social (24 preguntas) realizando primero un estudio exploratorio y confirmatorio con una muestra de 859 estudiantes mujeres de sureste de Estados Unidos. Posteriormente fue revisada esta estructura con muestras femeninas estadunidenses del sureste $(n=440)$, costa este $(n=304)$ y norte medio $(n=$ 349), así como muestras de Italia, Inglaterra y Australia, proponiendo una estructura de 22 preguntas en cinco factores: 1) Delgadez/Baja Grasa Corporal y 2) Muscularidad/Atlético para el rubro Internalización y 3) Familia, 4) Pares y 5) Medios para el rubro Presión. Quedó pendiente constatar la estructura para varones.

Posteriormente Schaefer, Harrieger, Heinberg, Soderberg y Thompson en 2017 realizaron una revisión al SATAQ-4, para tener la versión SATAQ- 4R, en la cual se propuso:

a) Valorar preguntas de internalización de ideal muscular y revisar si se eliminaba la referencia a físico "atlético" y quedarse sólo con el deseo de muscularidad. b) Conscientes de aceptar definiciones de internacionalización basadas en comportamientos, se pretende incluir los ítems que estén más enfocados en aspectos cognitivos de la internalización.

c) Incluir preguntas que se centraran en dimensiones generales de apariencia (no especificando delgadez/baja grasa corporal o muscularidad) ya que había evidencias que algunas preguntas, formaban este factor.

d) Finalmente, la inclusión de preguntas de la presión por la muscularidad, permite valorar esta situación más importante en el caso de los varones.

La versión del SATAQ-4R contiene 31 preguntas para mujeres y 28 para varones, para ambas versiones se propuso una estructura de siete factores y sólo en el caso de las mujeres se realizó su análisis factorial confirmatorio, lo cual puede verse en la tabla 7. Esta versión tendrá que ser probada nuevamente, ya que deja algunas cuestiones pendientes, como probar el análisis confirmatorio en muestra de varones.

\section{Conclusión}

La importancia de valorar las influencias socioculturales en la interiorización de los modelos estéticos corporales es relevante, porque a través de los medios masivos de comunicación, la familia, los amigos y el

Tabla 7. Características psicométricas del SATAQ-4 y SATAQ-4R.

\begin{tabular}{lll} 
Autores, año y país & Participantes & Resultados \\
\hline Schaefer et al. (2015) & $1^{\text {a } \text { Estudio } 859 \text { mujeres estudiantes }}$ & Se propone una estructura de 22 preguntas en cinco factores: \\
Estados Unidos, Italia, & universitarias del sureste de & Internalización \\
Inglaterra y Australia. & Estados Unidos. & Delgadez/Baja grasa corporal Alfa $=.87$ \\
& $2^{\text {a Estudio }}$ & Muscularidad/Atlético Alfa $=.91$ \\
& Costa este $(n=440)$, Costa oeste $(n$ & Presión \\
& $=304)$ y norte medio $(n=349)$ de & Familia Alfa $=.90$ \\
& Estados Unidos. & Pares $A l f a=.90$ \\
& 362 mujeres estudiantes & Medios $A l f a=.95$ \\
& universitarias de Italia, Inglaterra & Alfa total $=.93$ \\
& y Australia. & Análisis confirmatorio: $R M S E A=.07, S R M R=.05, T L I=.973 y$ \\
& $C F I=.976$
\end{tabular}




\section{Tabla 7 (Continuación). Características psicométricas del SATAQ-4 y SATAQ-4R.}

\begin{tabular}{|c|c|c|}
\hline Autores, año y país & Participantes & Resultados \\
\hline $\begin{array}{l}\text { Llorente, Cleaves, } \\
\text { Warren, Pérez-de-Eulate y } \\
\text { Rakhkovskaya (2015) } \\
\text { España }\end{array}$ & $\begin{array}{l}260 \text { Mujeres universitarias del País } \\
\text { Vasco, con un promedio de edad } \\
\text { de } 20.46(D E=2.59)\end{array}$ & $\begin{array}{l}\text { El análisis factorial indicó los siguientes factores: } \\
\text { Internacionalización } \\
\text { Delgado Alfa }=.90 \\
\text { Muscular } A l f a=.89 \\
\text { Presión } \\
\text { Familia Alfa }=.88 \\
\text { Iguales Alfa }=.94 \\
\text { Medios Alfa }=.97 \\
\text { Alfa total: }=.93 \\
\text { Análisis factorial confirmatorio: } R M S E A=.08, S R M R=.07, T L I \\
=.97 \text { y } C F I=.98\end{array}$ \\
\hline $\begin{array}{l}\text { Rodgers et al. (2016) } \\
\text { Francia }\end{array}$ & $\begin{array}{l}1^{\circ} \text { muestra } 207 \text { estudiantes } \\
\text { mujeres de Universidad, } M=21.69 \\
(D E=4.10) \\
2^{\circ} \text { muestra } 202 \text { estudiantes } \\
\text { mujeres } M=21.89(D E=5.28)\end{array}$ & $\begin{array}{l}\text { El análisis factorial indicó los siguientes factores: } \\
\text { Internacionalización: } \\
\text { 1.- Delgado/baja-grasa-corporal, Alfa = } .84 \text { a } .89 \\
\text { 2.- Muscular/Atlético, Alfa }=.92 \\
\text { Presión }\end{array}$ \\
\hline $\begin{array}{l}\text { Yamamiya et al. (2016) } \\
\text { Japón }\end{array}$ & $\begin{array}{l}948 \text { mujeres, en escuelas públicas } \\
\text { de Japón } \\
\text { M de edad }=16.23(D E=0.69)\end{array}$ & $\begin{array}{l}\text { Se reduce a } 20 \text { preguntas pero se conserva la estructura de } \\
\text { cinco factores: } \\
\text { Internacionalización: } \\
\text { 1.- Delgado/baja-grasa-corporal, } \\
\text { Alfa }=.78 \\
\text { 2.- Muscular/Atlético Alfa }=.82 \\
\text { Presión } \\
\text { 3.- Familia Alfa }=.91 \\
\text { 4.- Iguales Alfa }=.95 \\
\text { 5.- Medios Alfa }=.96 \\
\text { Alfa total = .93 } \\
\text { Análisis factorial confirmatorio: } R M S E A=.07, S R M R=.04 \text { y } \\
C F I=.95 \\
\text { Se aclara que se pueden conservar las dos preguntas, por el } \\
\text { análisis de validez confirmatorio }\end{array}$ \\
\hline $\begin{array}{l}\text { Schaefer et al. (2017) } \\
\text { Estados Unidos }\end{array}$ & $\begin{array}{l}1^{\circ} \text { estudio } 1,114 \text { estudiantes } \\
\text { mujeres } \\
2^{\circ} \text { estudio } 275 \text { estudiantes mujeres } \\
3^{\circ} 290 \text { estudiantes varones }\end{array}$ & $\begin{array}{l}\text { Se proponen para mujeres } 31 \text { preguntas y } 28 \text { preguntas para } \\
\text { varones, todo en una estructura de } 6 \text { factores, se presenta su } \\
\text { Alfa mujeres/varones. } \\
\text { Internacionalización } \\
\text { Delgado/cuerpo bajo en grasa Alfa }=.83 / 75 \\
\text { Muscular Alfa }=.82 / 87 \\
\text { Atractivo general Alfa }=.80 / 83 \\
\text { Presión } \\
\text { Familia, Alfa }=.84 / .89 \\
\text { Iguales } A l f a=.92 / .91 \\
\text { Medios Alfa }=.94 / .96 \\
\text { Análisis confirmatorio sólo con las muestras de mujeres. } \\
\text { RMSEA }=.07, S R M R=.05 \text { y } C F I=.91\end{array}$ \\
\hline
\end{tabular}


entorno social se transmiten valores sobre "lo corporal" y la apreciación de la belleza.

El desafió actual es grande, porque socioculturalmente occidente cuenta con una gran preocupación por el cuerpo y la apariencia, lo cual se acentúa al existir un considerable estigma hacia la obesidad; por tanto, la vulnerabilidad ante las influencias socioculturales se vuelve un importante factor de riesgo para trastornos alimentarios y de la imagen corporal en estas sociedades, en las que está incluida Latinoamérica por supuesto.

Al revisar los dos cuestionados más usados en Hispanoamérica, es interesante observar que se construyeron o mejor dicho se publicaron en tiempos muy semejantes; 1994 el CIMEC con 40 preguntas, mientras 1995 el SATAQ con 14 preguntas. No obstante, parece ser más conocido internacionalmente el SATAQ en el caso de Hispanoamérica, podemos pensar que el CIMEC ha sido el más utilizado, sobre todo en México y España, de acuerdo con la búsqueda realizada.

Los procesos de difusión y validación a lo largo de estos 20 años también son diferentes, y eso tiene que ver con su construcción original y su difusión. En el caso del CIMEC podemos decir que ha trabajado principalmente en reducir el número de preguntas, debido a que desde su creación cuenta con aspectos de influencia de publicidad, de amigos y familia, también se crearon dos versiones diferentes para varones y mujeres desde un inicio, ya que se tiene una visión clara que los objetivos a perseguir son diferentes entre varones y mujeres. Su utilización en Latinoamérica no ha presentado problemas y al parecer las versiones de México han sido adecuadas, cierto es que tampoco hay muchos datos de validación y no existen evidencias de transformar el instrumento, quizás se deba a su buen funcionamiento.

En el caso del SATAQ su creación y primera versión se centró en las influencias de los medios para mujeres, por lo que en cada versión se fue aumentando las influencias de los pares, familia, así como los modelos estéticos de musculatura para los varones. De las 14 preguntas originales ahora en su versión más difundida SATAQ-3 se tienen 22 preguntas, y en la versión del SATAQ-4R, se proponen 31 preguntas para mujeres y 28 para varones. La revisión de sus propiedades psicométricas es constante y tiene que ver con otras culturas, como la asiática y árabe, por lo que es claro que su difusión es mayor al CIMEC. Por los estudios revisados podemos decir que los dos instrumentos cumplen con su tarea de valorar las influencias de los modelos estéticos corporales.

\section{Referencias}

Alvarez, G., Franco, K., López, X., Mancilla, J., y Vázquez, R. (2009). Imagen corporal y trastornos de la conducta alimentaria. Revista de Salud Pública, 11, 4, 568-578. https:// doi.org/10.1590/S0124-00642009000400008.

Amaya, A., Mancilla-Diaz, J. M., Álvarez, G. L., Ortega, M., Larios, M., y Martínez, J. I. (2013). Edad, consciencia e interiorización del ideal corporal como predictores de insatisfacción y conductas alimentarias anómalas. Revista Colombiana de Psicología, 22(1), 121-133.

Barrientos, N., Escoto, C., Bosques, L. Enríquez, J., y Juárez, C. (2014). Interiorización de ideales estéticos y preocupación corporal en hombres y mujeres usuarios de gimnasio. Revista Mexicana de Trastornos Alimentarios, 5(1), 29-38.

Cash, T. F., y Pruzinsky, T. (2002). Body Images: A Handbook of Theory, Research, and Clinical Practice. Nueva York: Guilford Press. https://doi.org/10.1080/10640260390218738

Cruz, R., Ávila, M., Velázquez, H, Castillo, E., y Francis, D. (2013). Evaluación de factores de riesgo de TCA en estudiantes de nutrición. Revista Mexicana de Trastornos Alimentarios, 4(1), 37-44.

Cusumano, D.L., y Thompson, J.K. (1997). Body image and body shape ideals in magazines: Exposure, awareness, and internalization. Sex Roles, 37, 701-721. https://doi. .org/10.1007/BF02936336.

Dunker, K. L. L., Fernandes, C. P. B., y Carreira, F. D. (2009). Influência do nível socioeconômico sobre comportamentos de risco para transtornos alimentares em adolescentes. Jornal Brasileiro de Psiquiatria, 58(3), 156-161. https://doi.org/10.1590/S0047-20852009000300003.

Escoto, M., Camacho, E., y Mancilla, J. (2010). Risk factors for the development of symptomatology of eating disorders: prospective study. Journal of Behavior, Health \& Social Issues, 2(1), 7-15. https://doi.org/10.5460/jbhsi. v2.i1.02.

Esnaola, I., Rodríguez, A., y Goñi, A. (2010). Body dissatisfaction and perceived sociocultural pressures: gender and age differences. Salud Mental, 33, 21-29.

Espósito, D. (2015). Influencia del modelo estético corporal prevalente e insatisfacción con la imagen corporal en 
estudiantes de escuela secundaria básica. Revista Mexicana de Trastornos Alimentarios, 6(2), 91-96. https://doi. org/10.1016/j.rmta.2015.08.001.

Fortes, L. S., Meireles, J. F. F., Paes, S. T., Dias, F. C., Cipriani, F.M., y Ferreira, M. E. C. (2015). An association between the internalization of body image, depressive symptoms and restrictive eating habits among young males. Ciência \& Saúde Coletiva, 20(11), 3457-3465. https:// doi.org/10.1590/1413-812320152011.00152015.

Franco, K., Díaz, F., López, A., Escoto, M., y Jaime, E. (2013). Variables predictoras de riesgo de trastorno del comportamiento alimentario en mujeres. Terapia Psicológica, 31(2), 219-225. https://doi. org/10.4067/S0718-48082013000200008.

Galioto, R., y Crowther, J.H. (2013). The effects of exposure to slender and muscular images on male body dissatisfaction. Body Image, 10(4), 566-573. https://doi. org/10.1016/j.bodyim.2013.07.009.

Harrison, K., y Hefner, V. (2006). Media exposure, current and future body ideals, and disordered eating among preadolescent girls: a longitudinal panel study. Journal of Youth and Adolescence, 35, 146-156. https://doi. org/10.1007/s10964-005-9008-3.

Heinberg, L., Thompson, K.L., y Stormer, S. (1995). Development and validation of the sociocultural attitudes towards appearance questionnaire. International Journal of Eating Disorders, 17(1), 81-89. https:// doi.org/10.1002/1098-108X(199501)17:1<81::AID -EAT2260170111>3.0.CO;2-Y.

Hernández, N., Alves, D., Arroyo, M., y Basabe, N. (2012). Del miedo a la obesidad a la obsesión por la delgadez; actitudes y dieta. Nutrición Hospitalaria, 27(4), 1148-1155. https://doi.org/10.3305/nh.2012.27.4.5829.

Jackson, T., y Chen, H. (2010). Factor structure of the Sociocultural Attitudes Towards Appearance Questionnaire-3 (SATAQ-3) among adolescent boys in China. Body Image, 7, 439-355. https://doi.org/10.1016/j. bodyim.2010.07.003.

Jáuregui, I., Tomillo, S., Santiago, M., y Bolaños, P. (2011). Body shape model, physical activity and eating behavior. Nutrición Hospitalaria, 26(1), 201-207. https://doi. .org/10.3305/nh.2011.26.1.4637.

Leit, R. A., Gray, J. J., y Pope, H. G. Jr. (2002). The media's representation of the ideal male body: a cause for muscle dysmorphia? International Journal of Eating Disorders, 31(3), 334-338. https://doi.org/10.1002/eat.10019.

Llorente, E., Gleaves, D. H., Cortney S. Warren, C.S., Pérez-de-Eulate, L., y Rakhkovskaya, L. (2015). Translation and validation of a spanish version of the sociocultural attitudes towards appearance questionnaire-4 (SATAQ-4). International Journal of Eating Disorders, 48, 170-175. https://doi.org/10.1002/eat.22263.
Madanat, H. N., Hawks, S. R., y Brown, R. B. (2006). Validation of the Sociocultural Attitudes Towards Appearance Questionnaire-3among a random sample of Jordanian women. Body Image, 3(4), 421-425. https:// doi.org/10.1016/j.bodyim.2006.09.004.

Magallares, A. (2013). Social risk factors related to eating disorders in women. Revista Latinoamericana de Psicología, 45(1), 147-154

Magallares, A. (2016). Eating concerns, body dissatisfaction, thinness internalization and antifat attitudes and their relationship with gender ideology in a sample of men. Anales de Psicología, 32(1), 167-173. https://doi. org/10.6018/analesps.32.1.182651.

Mancilla, J., Lameiras, M., Vázquez, R., Álvarez, G., Franco, K., López, X., y Ocampo, M. (2010). Influencias socioculturales y conductas alimentarias no saludables en hombres y mujeres de España y México. Revista Mexicana de Trastornos Alimentarios, 1(1), 36-47.

Mancilla, J., López, X., Franco, K., Álvarez, G., Vázquez, R., Ocampo, M., y Amaya, A. (2012). Role of Peer Influence and Thin-ideal Internalization on Body Dissatisfaction and Disordered Eating in Mexican Girls. Revista Colombiana de Psicología, 21(2), 341-351.

Mockdece, C., Fernandes, J., Berbert, P. H., Sousa, S., y Caputo, M. E. (2016). Body dissatisfaction among artistic gymnastics adolescent athletes and non-athletes. Revista Brasileira de Cineantropometria \& Desempenho Humano, 18(1), 82-92. https://doi.org/10.5007/1980-0037.20 16v18n1p82.

Murawski, B., Elizathe, L., Custodio, J., y Rutsztein, G. (2015). Argentine validation of the Sociocultural Attitudes Towards Appearance Questionnaire-3. Revista Mexicana de Trastornos Alimentarios, 6, 73-90. https://doi. .org/10.1016/j.rmta.2015.09.001.

Rivarola, M., y Penna, F. (2006). Los factores socioculturales y su relación con los trastornos alimentarios e imagen corporal. Revista Intercontinental de Psicología y Educación, 8, 2, 61-72.

Rodgers, R. F., Schaefer, L. M., Thompson, J. K., Girard, M., Bertrand, M., y Chabrol, H. (2016). Psychometric properties of the Sociocultural Attitudes Towards Appearance Questionnaire-4 (SATAQ-4) in French women and men. Body Image, 17, 143-151. https://doi.org/ 10.1016/j.bodyim.2016.03.002.

Rodríguez, S., y Cruz, S. (2008). Insatisfacción corporal en adolescentes latinoamericanas y españolas. Psicothema, 20, 1, 131-137.

Rousseau, A., Valls, M., y Chabrol, H. (2010). The Sociocultu-ral Attitudes Towards Appearance Scale-3 (SATAQ-3): Étude de validation de la version franc ${ }_{3}$ aise. L'Encéphale, 36(4), 270-276. https://doi.org/10.1016/j. encep.2008.03.013. 
Ruiz, A., Vázquez, R., Mancilla, J. y Trujillo, E. (2010). Factores socioculturales de hijas y padres en los trastornos del comportamiento alimentario. Enseñanza e Investigación en Psicología, 15(2), 311-324.

Schaefer, L. M., Burke, N. L., Thompson, J. K., Dedrick, Heinberg, L. J., Calogero, R. M., ... Swami, V. (2015). Development and validation of the sociocultural attitudes towards appearance questionnaire-4 (SATAQ-4). Psychological Assessment, 27(1), 54-67. https:/doi. org/10.1037/a0037917.

Schaefer, L. M., Harrieger, J. A., Heinberg, L. J., Soderberg, T., y Thompson, J. K. (2017). Development and Validation of the Sociocultural Attitudes Towards Appearance Questionnaire-4-Revised (SATAQ-4R). International Journal of Eating Disorders, 50(2), 104-117. https://doi. org/10.1002/eat.22590

Soares,A.C.,Conti,M.A.,Caputo,M.E.,yFilgueiras,J.F.(2015) Avaliação psicométrica do questionário de atitudes socioculturais em relação à aparência-3 (SATAQ-3 para adolescentes. Psicología Teoría e Pesquisa, 31(4), 471-479. https://doi.org/10.1590/0102-37722015042419471479.

Stefanile, C., Matera, C., Nerini, A., y Pisani, E. (2009). Validation of an Italian version of the Sociocultural Attitudes Towards Appearance Questionnaire-3 (SATAQ-3) on adolescent girls. Body Image, 8(4), 432-436. https://doi.org/10.1016/j.bodyim.2011.06.001.

Stice, E., Schupak-Neuberg, E., Shaw, H.E., y Stein, R.I. (1994). Relation of media exposure to eating disorder symptomatology: An examination of mediating mechanisms. Journal of Abnormal Psychology, 103, 836-840. https://doi.org/10.1037/0021-843X.103.4.836.

Thompson, J. K., Heinberg, L. J., Altabe, M., y Tantleff-Dunn, S. (1999). Exacting beauty: Theory, assessment, and treatment of body image disturbance. Washington, DC: American Psychological Association. https://doi. org/10.1037/10312-000.

Thompson, J. K., van den Berg, P., Roehrig M, Guarda, A. S., y Heinberg, L. J. (2004). The sociocultural attitudes towards appearance scale-3 (SATAQ-3): development and validation. International Journal of Eating Disorders, 35(3), 293-304. https://doi.org/10.1002/eat.10257.

Tiggemann, M. (2014) The status of media effects on body image research: commentary on articles in the themed issue on body image and media. Media Psychology, 17(2), 127-133, https://doi.org/10.1080/15213269.2014.891822.

Toro, J., Castro, J., Gila, A., y Pombo, C (2005). Assessment of sociocultural influences on the body shape model in adolescent males with anorexia nervosa. European Eating Disorders Review, 13, 351-359. https://doi. org/10.1002/erv.650.

Toro, J., Salamero, M., y Martinez, E. (1994). Assessment of sociocultural influences on the aesthetic body shape model in anorexia nervosa. Acta Psychiatrica Scandinavica, 89(3), 147-151. https://doi.org/10.1111/j.1600-0447.1994. tbo8084.x.

Van den Berg, P., Thompson, J.K., Obremski-Brandon, K., y Coovert, M. (2002). The Tripartite Influence model of body image and eating disturbance: A covariance structure modeling investigation testing the mediational role of appearance comparison. Journal of Psychosomatic Research, 53(5), 1007-1020. https://doi.org/10.1016/ So022-3999(02)00499-3.

Vázquez, R., Alvarez, R., y Mancilla, J. M. (2000). Consistencia interna y estructura factorial del Cuestionario de Influencia de los Modelos Estéticos Corporales (CIMEC), en población mexicana. Salud Mental, 23(6), 18-24.

Vázquez, R., López, X., Álvarez, G., Mancilla, J., y Ruíz, A. O. (2006). Insatisfacción corporal e influencia de los modelos estéticos en niños y jóvenes varones mexicanos. Enseñanza e Investigación en Psicología, 11(1), 185-197.

Vázquez, R., Mancilla, J. M., Mateo, C., López, X., Álvarez, G. L., y Ruiz, A. O. (2005). Trastornos del comportamiento alimentario y factores de riesgo en una muestra incidental de jóvenes mexicanos. Revista Mexicana de Psicología, 22, 53-63.

Vázquez, R., Velázquez, G., López, X., Álvarez, G., Mancilla, J. M., y Franco, K. (2010). Consistencia interna y estructura factorial del cuestionario de influencias del modelo estético corporal (CIMEC) en estudiantes mexicanos. Revista Psicología y Ciencia Social, 12(1), 5-11.

Yamamiya, Y., Shimai, S., Schaefer, L.M., Thompson, J.K., Shroff, H., Sharma, R., y Ordaz, D.L. (2016). Psychometric properties and validation of the Sociocultural Attitudes Towards Appearance Questionnaire-4 (SATAQ-4) with a sample of Japanese adolescent girls. Body Image, 19, 89-97. https://doi.org/10.1016/j. bodyim.2016.08.006.

Young, A., Gabriel, S., y Hollar, J. (2013). Batman to the rescue! The protective effects of parasocial relationships with muscular superheroes on men's body image. Journal of Experimental Social Psychology, 49, 173-177. https:// doi.org/10.1016/j.jesp.2012.08.003 